\title{
POLARISATION DE FONCTIONS SYMÉTRIQUES
}

par

Mohamed Krir

Résumé. - L'objet de cet article est de donner une preuve d'un résultat de Lønsted et Kleiman énoncé et non démontré dans $[\mathbf{L} \varnothing \mathbf{K l}]$. On donne une décomposition algébrique de certaines fonctions symétriques de multi-ensembles d'indéterminées.

\begin{abstract}
The aim of this article is to prove a result of Lønsted et Kleiman, stated without proof in $[\mathbf{L} \varnothing \mathbf{K} \mathbf{l}]$. We give algebraic decomposition of certain symmetric functions on finite set of $n$-tuples of variables.
\end{abstract}

\section{Introduction}

Soit $R$ un anneau commutatif, $A$ une $R$-algèbre et $G$ un groupe fini de $R$-automorphismes de $A$. La sous $R$-algèbre de $A$ invariante par $G$ est notée $A^{G}$. Lønsted et Kleiman ont introduit dans $[\mathbf{L} \varnothing \mathbf{K l} \mathbf{l}]$ une sous $R$-algèbre $\Sigma_{R}^{G}(A)$ de $A^{G}:$ si $G=\left\{g_{1}, g_{2}, \ldots, g_{n}\right\}$ et si on pose, pour tout $a \in A$ et pour tout entier $j$ tel que $1 \leq j \leq n, \sigma_{j}(a)=S_{j}\left(g_{1} a, \ldots, g_{n} a\right)$ où $S_{j}$ est la $j$-ième fonction symétrique élémentaire en $n$ variables alors $\Sigma_{R}^{G}(A)=R\left[\bigcup_{1 \leq j \leq n} \sigma_{j}(A)\right]$.

Dans [BeMé1], Bertin et Mézard valident cette construction dans le cas où $G$ est un schéma en groupe fini et plat et étudient les propriétés de l'algèbre $\Sigma_{R}^{G}(A)$ ainsi associée. Cette construction de $\Sigma_{R}^{G}(A)$ se comporte de façon fonctorielle par changement de base et par passage au quotient. Elle permet une approche combinatoire des invariants tout à fait adaptée à la géométrie algébrique. Elle est valide pour l'action sous un groupoïde fini et plat [DeGa] et [Gi]. Elle est adaptée à la théorie des déformations $G$-équivariantes [BeMé2]. Elle est également adaptée à l'étude locale des quotients de l'action d'un groupe fini d'une courbe semi-stable et à la description de la structure du groupe d'inertie au point double [Ra].

L'objet de cet article est d'expliciter des relations algébriques entre certaines fonctions symétriques et notamment de donner une démonstration complète du résultat énoncé au lemme 4.5 de $[\mathbf{L} \varnothing \mathbf{K} \mathbf{l}]$ et non démontré.

Classification mathématique par sujets (2000). — $05 \mathrm{E} 05$.

Mots clefs. - Fonction symétrique, fonction symétrique élémentaire, décomposition algébrique. 
Dans une première partie on introduit les notations et conventions utilisées et on définit la notion de fonctions symétriques polarisées. Ces fonctions sont $\mathfrak{S}$-invariantes où $\mathfrak{S}$ est un produit de groupes symétriques; dans ce sens, elles généralisent la notion de fonctions symétriques. La deuxième partie est réservée à la preuve de résultats de décomposition algébrique des fonctions symétriques et des fonctions symétriques polarisées. Dans la dernière partie, on démontre le résultat principal.

\section{Notations et conventions}

Dans cette partie on introduit les notations utilisées dans toute la suite et on définit la notion de fonctions symétriques polarisées.

Tous les polynômes considérés dans cet article sont à coefficients dans un anneau commutatif unitaire. Soit $n \in \mathbb{N}^{*}$ on note $\llbracket 1, n \rrbracket$, l'ensemble des entiers compris entre 1 et $n$. Soit $k \in \llbracket 1, n \rrbracket$, la $k$-ième fonction symétrique élémentaire en $n$ indéterminées indépendantes $\left(x_{1}, \ldots, x_{n}\right)$ est par définition (c.f. par exemple [Ma] pour plus de détails),

$$
S_{k}\left(x_{1}, \ldots, x_{n}\right)=\sum_{1 \leq i_{1}<i_{2}<\cdots<i_{k} \leq n} x_{i_{1}} x_{i_{2}} \cdots x_{i_{k}}
$$

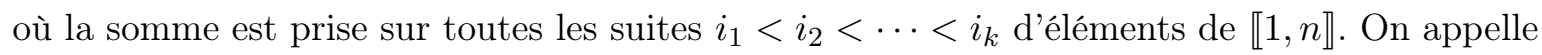
support du monôme $x_{i_{1}} x_{i_{2}} \cdots x_{i_{k}}$ l'ensemble

$$
\operatorname{Supp}\left(x_{i_{1}} x_{i_{2}} \cdots x_{i_{k}}\right):=\left\{i_{1}, \ldots, i_{k}\right\}
$$

Soit $q \in \mathbb{N}^{*}$. On considère $q$ ensembles $X^{(1)}, \ldots, X^{(q)}$ de $n$ variables. On pose, pour tout $\ell \in \llbracket 1, q \rrbracket$,

$$
X^{(\ell)}=\left(x_{1}^{(\ell)}, \ldots, x_{n}^{(\ell)}\right)
$$

et on définit la somme des $\left(X^{(i)}\right)_{1 \leq i \leq q}$ par :

$$
X^{(1)}+\cdots+X^{(q)}=\left(x_{1}^{(1)}+\cdots+x_{1}^{(q)}, \ldots, x_{n}^{(1)}+\cdots+x_{n}^{(q)}\right) .
$$

Pour tout $\left(r_{1}, \ldots, r_{q}\right) \in \mathbb{N}^{q}$, on définit formellement le monôme

$$
\left(X^{(1)}\right)^{r_{1}} \cdots\left(X^{(q)}\right)^{r_{q}}
$$

en les variables $X^{(1)}, \ldots, X^{(q)}$ par

$$
\left(X^{(1)}\right)^{r_{1}} \cdots\left(X^{(q)}\right)^{r_{q}}=\left(\left(x_{1}^{(1)}\right)^{r_{1}} \cdots\left(x_{1}^{(q)}\right)^{r_{q}}, \ldots,\left(x_{n}^{(1)}\right)^{r_{1}} \cdots\left(x_{n}^{(q)}\right)^{r_{q}}\right) .
$$

Pour tout $q$-uplet $\left(\alpha_{1}, \ldots, \alpha_{q}\right) \in \mathbb{N}^{q}$ tel que $\alpha_{1}+\cdots+\alpha_{q} \leq n$, on pose

$$
S_{\alpha_{1}, \cdots, \alpha_{q}}\left(X^{(1)}, \ldots, X^{(q)}\right)=\sum\left(x_{i_{1}^{(1)}}^{(1)} \cdots x_{i_{\alpha_{1}}^{(1)}}^{(1)}\right) \cdots\left(x_{i_{1}^{(q)}}^{(q)} \cdots x_{i_{\alpha_{q}}^{(q)}}^{(q)}\right)
$$

où la somme est prise sur toutes les suites disjointes partiellement ordonnées

$$
\left(i_{1}^{(k)}<\cdots<i_{\alpha_{k}}^{(k)}\right)_{1 \leq k \leq q}
$$


d'éléments de $\llbracket 1, n \rrbracket$, i.e. tels que les $q$ supports $\left(\operatorname{Supp}\left(x_{i_{1}^{(k)}}^{(k)} \cdots x_{i_{\alpha_{k}}^{(k)}}^{(k)}\right)\right)_{1 \leq k \leq q}$ soient deux à deux disjoints.

Définition 1.1. - Les fonctions définies par (1) sont appelées fonctions symétriques polarisées.

Remarque 1.2. - Si $\alpha_{k}=0$ alors la variable $X^{(k)}$ n'intervient pas dans la somme (1) et si tous les $\alpha_{k}$ sont nuls sauf un (disons $\alpha_{i}$ ) alors

$$
S_{0, \ldots, 0, \alpha_{i}, 0, \ldots, 0}=S_{\alpha_{i}}\left(X^{(i)}\right)
$$

Exemple 1.3. - Pour $q=3, n=5,\left(\alpha_{1}, \alpha_{2}, \alpha_{3}\right)=(1,1,2)$, on pose, pour alléger les notations,

$$
\begin{aligned}
& X^{(1)}=X=\left(x_{1}, x_{2}, x_{3}, x_{4}, x_{5}\right) \\
& X^{(2)}=Y=\left(y_{1}, y_{2}, y_{3}, y_{4}, y_{5}\right) \\
& X^{(3)}=Z=\left(z_{1}, z_{2}, z_{3}, z_{4}, z_{5}\right)
\end{aligned}
$$

on a alors

$$
\begin{aligned}
S_{1,1,2}(X, Y, Z) & =\left(x_{1} y_{2}+x_{2} y_{1}\right)\left(z_{3} z_{4}+z_{3} z_{5}+z_{4} z_{5}\right) \\
& +\left(x_{1} y_{3}+x_{3} y_{1}\right)\left(z_{2} z_{4}+z_{2} z_{5}+z_{4} z_{5}\right) \\
& +\left(x_{1} y_{4}+x_{4} y_{1}\right)\left(z_{2} z_{3}+z_{2} z_{5}+z_{3} z_{5}\right) \\
& +\left(x_{1} y_{5}+x_{5} y_{1}\right)\left(z_{2} z_{3}+z_{2} z_{4}+z_{3} z_{4}\right) \\
& +\left(x_{2} y_{3}+x_{3} y_{2}\right)\left(z_{1} z_{4}+z_{1} z_{5}+z_{4} z_{5}\right) \\
& +\left(x_{2} y_{4}+x_{4} y_{2}\right)\left(z_{1} z_{3}+z_{1} z_{5}+z_{3} z_{5}\right) \\
& +\left(x_{2} y_{5}+x_{5} y_{2}\right)\left(z_{1} z_{3}+z_{1} z_{4}+z_{3} z_{4}\right) \\
& +\left(x_{3} y_{4}+x_{4} y_{3}\right)\left(z_{1} z_{2}+z_{1} z_{5}+z_{2} z_{5}\right) \\
& +\left(x_{3} y_{5}+x_{5} y_{3}\right)\left(z_{1} z_{2}+z_{1} z_{4}+z_{2} z_{4}\right) \\
& +\left(x_{4} y_{5}+x_{5} y_{4}\right)\left(z_{1} z_{2}+z_{1} z_{3}+z_{2} z_{3}\right)
\end{aligned}
$$

Remarque 1.4. - Notons $\mathfrak{S}_{\alpha_{k}}$ le $\alpha_{k}$-ième groupe symétrique et posons

$$
\mathfrak{S}=\mathfrak{S}_{\alpha_{1}} \times \cdots \times \mathfrak{S}_{\alpha_{q}} .
$$

Soit $\tau=\tau_{1} \times \cdots \times \tau_{k} \in \mathfrak{S}$. On fait agir $\tau$ sur $S_{\alpha_{1}, \cdots, \alpha_{q}}\left(X^{(1)}, \ldots, X^{(q)}\right)$ en faisant agir $\tau_{k}$ sur chaque monôme de la forme $\left(x_{i_{1}^{(k)}}^{(k)} \cdots x_{i_{\alpha_{k}}^{(k)}}^{(k)}\right)$. Comme cette dernière action consiste à permuter les indéterminées alors la fonction $S_{\alpha_{1}, \cdots, \alpha_{q}}\left(X^{(1)}, \ldots, X^{(q)}\right)$ est invariante par $\mathfrak{S}$.

\section{Décomposition algébrique des fonctions symétriques}

Dans ce paragraphe on montre trois lemmes. Dans le premier, on exprime une certaine fonction symétrique élémentaire comme somme de fonctions symétriques polarisées de la forme (1) et dans les suivants, on décompose le produit de fonctions symétriques élémentaires en une somme de fonctions symétriques polarisées. 
Lemme 2.1. - Soit $q, n \in \mathbb{N}^{*}$ et soit $X^{(\ell)}=\left(x_{1}^{(\ell)}, \ldots, x_{n}^{(\ell)}\right)$ pour $1 \leq \ell \leq q$, q ensembles de $n$ variables. Pour tout entier $j \leq n$, on a

$$
S_{j}\left(X^{(1)}+\cdots+X^{(q)}\right)=\sum_{\alpha_{1}+\cdots+\alpha_{q}=j} S_{\alpha_{1}, \ldots, \alpha_{q}}\left(X^{(1)}, \ldots, X^{(q)}\right)
$$

Démonstration. - Ecrivons $X^{(1)}+\cdots+X^{(q)}=\left(Y_{1}, \ldots, Y_{n}\right)$ où $Y_{k}=x_{k}^{(1)}+\cdots+x_{k}^{(q)}$ pour tout $k \in \llbracket 1, n \rrbracket$. On a donc pour $j \leq n$,

$$
\begin{aligned}
S_{j}\left(X^{(1)}+\cdots+X^{(q)}\right) & =S_{j}\left(Y_{1}, \cdots, Y_{n}\right) \\
& =\sum_{1 \leq i_{1}<i_{2}<\cdots<i_{j} \leq n} Y_{i_{1}} Y_{i_{2}} \cdots Y_{i_{j}} \\
& =\sum_{1 \leq i_{1}<i_{2}<\cdots<i_{j} \leq n}\left[\left(\sum_{r=1}^{q} x_{i_{1}}^{(r)}\right) \cdots\left(\sum_{r=1}^{q} x_{i_{j}}^{(r)}\right)\right]
\end{aligned}
$$

Chaque produit $\left[\left(\sum_{r=1}^{q} x_{i_{1}}^{(r)}\right) \cdots\left(\sum_{r=1}^{q} x_{i_{j}}^{(r)}\right)\right]$ est la somme de $q^{j}$ termes, chacun d'entre eux faisant intervenir, pour chaque entier $k \in \llbracket 1, n \rrbracket, \alpha_{k}$ composantes de $X^{(k)}$ et est donc de la forme

avec

$$
\left(x_{i_{1}^{(1)}}^{(1)} \cdots x_{i_{\alpha_{1}}^{(1)}}^{(1)}\right) \cdots\left(x_{i_{1}^{(q)}}^{(q)} \cdots x_{i_{\alpha_{q}}^{(q)}}^{(q)}\right)
$$

$$
\begin{cases}\alpha_{1}+\cdots+\alpha_{q}=j & \\ 0 \leq \alpha_{k} \leq j & \text { pour } k=1, \ldots, q \\ i_{1}^{(k)}<\cdots<i_{\alpha_{k}}^{(k)} & \text { pour } k=1, \ldots, q\end{cases}
$$

et où les $q$ supports $\left(\operatorname{Supp}\left(x_{i_{1}^{(k)}}^{(k)} \cdots x_{i_{\alpha_{k}}^{(k)}}^{(k)}\right)\right)_{1 \leq k \leq q}$ sont deux à deux disjoints. Il s'ensuit qu'en sommant sur toutes les suites $i_{1}<\cdots<i_{j}$ de $\llbracket 1, n \rrbracket$, les produits

$$
\left[\left(\sum_{r=1}^{q} x_{i_{1}}^{(r)}\right) \cdots\left(\sum_{r=1}^{q} x_{i_{j}}^{(r)}\right)\right]
$$

on aura

$$
\begin{aligned}
S_{j}\left(X^{(1)}+\cdots+X^{(q)}\right) & =\sum_{\alpha_{1}+\cdots+\alpha_{q}=j} \sum\left(x_{i_{1}^{(1)}}^{(1)} \cdots x_{i_{\alpha_{1}}^{(1)}}^{(1)}\right) \cdots\left(x_{i_{1}^{(q)}}^{(q)} \cdots x_{i_{\alpha_{q}}^{(q)}}^{(q)}\right) \\
& =\sum_{\alpha_{1}+\cdots+\alpha_{q}=j} S_{\alpha_{1}, \ldots, \alpha_{q}}\left(X^{(1)}, \ldots, X^{(q)}\right)
\end{aligned}
$$

Lemme 2.2. - Soit $q, n \in \mathbb{N}^{*}$ et soit $X^{(\ell)}=\left(x_{1}^{(\ell)}, \ldots, x_{n}^{(\ell)}\right)$ pour $1 \leq \ell \leq q$, q ensembles de $n$ variables. Soit $\left(\alpha_{1}, \ldots, \alpha_{q}\right) \in \mathbb{N}^{q}$ tel que $\alpha_{1}+\cdots+\alpha_{q} \leq n$. Le produit $S_{\alpha_{1}}\left(X^{(1)}\right) \cdots S_{\alpha_{q}}\left(X^{(q)}\right)$ des fonctions symétriques élémentaires s'exprime comme somme de fonctions symétriques polarisées de la forme (1) dont les variables sont des monômes en $\left(X^{(i)}\right)$. 
Démonstration. — On calcule le produit des fonctions symétriques $S_{\alpha_{i}}\left(X^{(i)}\right)$.

$$
\prod_{i=1}^{q} S_{\alpha_{i}}\left(X^{(i)}\right)=\left(\sum_{i_{1}<\cdots<i_{\alpha_{1}}} x_{i_{1}}^{(1)} \cdots x_{i_{\alpha_{1}}}^{(1)}\right) \cdots\left(\sum_{i_{1}<\cdots<i_{\alpha_{q}}} x_{i_{1}}^{(q)} \cdots x_{i_{\alpha_{q}}}^{(q)}\right) .
$$

Ce produit est égal à une somme de termes, chacun étant un produit de $|\alpha|=\left(\alpha_{1}+\cdots+\alpha_{q}\right)$ indéterminées dont $\alpha_{k}$ indéterminées proviennent de $X^{(k)}$ pour $k=1, \ldots, q$. Ces différents termes sont des monômes à supports non disjoints.

Soit $\mathcal{P}$ l'ensemble des parties non vides de l'ensemble $\{1, \ldots, q\}$. Le cardinal de $\mathcal{P}$ est donc $N=2^{q}-1$. Pour tout $\sigma \in \mathcal{P}$ on définit le monôme $M_{\sigma}$ en les variables $X^{(i)}$ par

$$
M_{\sigma}=\prod_{i \in \sigma} X^{(i)}
$$

Pour chaque $N$-uplet $\left(j_{\sigma}\right)_{\sigma \in \mathcal{P}}$ d'entiers naturels vérifiant la condition

$$
\forall i \in\{1,2 \ldots, q\}, \sum_{\substack{\sigma \in \mathcal{P} \\ i \in \sigma}} j_{\sigma}=\alpha_{i}
$$

on considère la fonction symétrique polarisée

$$
S_{\left(j_{\sigma}\right)_{\sigma \in \mathcal{P}}}\left(\left(M_{\sigma}\right)_{\sigma \in \mathcal{P}}\right) .
$$

On a alors

$$
\prod_{i=1}^{q} S_{\alpha_{i}}\left(X^{(i)}\right)=\sum_{\left(j_{\sigma}\right)_{\sigma \in \mathcal{P}}} S_{\left(j_{\sigma}\right)_{\sigma \in \mathcal{P}}}\left(\left(M_{\sigma}\right)_{\sigma \in \mathcal{P}}\right)
$$

la somme étant prise sur tous les $N$-uplets $\left(j_{\sigma}\right)_{\sigma \in \mathcal{P}}$ vérifiant la condition (3).

Exemple 2.3. - Illustrons ce lemme 2.2 par un exemple où $n=5, q=3,\left(\alpha_{1}, \alpha_{2}, \alpha_{3}\right)=$ $(1,1,2)$. On a alors

$$
\begin{aligned}
S_{1}\left(X^{(1)}\right) S_{1}\left(X^{(2)}\right) S_{2}\left(X^{(3)}\right)= & \left(x_{1}^{(1)}+x_{2}^{(1)}+x_{3}^{(1)}+x_{4}^{(1)}+x_{5}^{(1)}\right) \\
& \left(x_{1}^{(2)}+x_{2}^{(2)}+x_{3}^{(2)}+x_{4}^{(2)}+x_{5}^{(2)}\right) \\
& \left(x_{1}^{(3)} x_{2}^{(3)}+x_{1}^{(3)} x_{3}^{(3)}+x_{1}^{(3)} x_{4}^{(3)}+x_{1}^{(3)} x_{5}^{(3)}+x_{2}^{(3)} x_{3}^{(3)}\right. \\
& \left.+x_{2}^{(3)} x_{4}^{(3)}+x_{2}^{(3)} x_{5}^{(3)}+x_{3}^{(3)} x_{4}^{(3)}+x_{3}^{(3)} x_{5}^{(3)}+x_{4}^{(3)} x_{5}^{(3)}\right)
\end{aligned}
$$

Ici $\mathcal{P}=\{\{1\},\{2\},\{3\},\{1,2\},\{1,3\},\{2,3\},\{1,2,3\}\}$ et la condition (3) se traduit par le système suivant :

$$
\left\{\begin{array}{l}
j_{\{1\}}+j_{\{1,2\}}+j_{\{1,3\}}+j_{\{1,2,3\}}=1 \\
j_{\{2\}}+j_{\{1,2\}}+j_{\{2,3\}}+j_{\{1,2,3\}}=1 \\
j_{\{3\}}+j_{\{1,3\}}+j_{\{2,3\}}+j_{\{1,2,3\}}=2
\end{array}\right.
$$

La situation est résumée dans le tableau suivant : 


\begin{tabular}{|c|c|c|c|c|c|c|c|}
\hline$\sigma$ & $\{1\}$ & $\{2\}$ & $\{3\}$ & $\{1,2\}$ & $\{1,3\}$ & $\{2,3\}$ & $\{1,2,3\}$ \\
\hline$M_{\sigma}$ & $X^{(1)}$ & $X^{(2)}$ & $X^{(3)}$ & $X^{(1)} X^{(2)}$ & $X^{(1)} X^{(3)}$ & $X^{(2)} X^{(3)}$ & $X^{(1)} X^{(2)} X^{(3)}$ \\
\hline$j_{\sigma}$ & 1 & 1 & 2 & 0 & 0 & 0 & 0 \\
\hline$j_{\sigma}$ & 1 & 0 & 1 & 0 & 0 & 1 & 0 \\
\hline$j_{\sigma}$ & 0 & 1 & 1 & 0 & 1 & 0 & 0 \\
\hline$j_{\sigma}$ & 0 & 0 & 2 & 1 & 0 & 0 & 0 \\
\hline$j_{\sigma}$ & 0 & 0 & 0 & 0 & 1 & 1 & 0 \\
\hline$j_{\sigma}$ & 0 & 0 & 1 & 0 & 0 & 0 & 1 \\
\hline
\end{tabular}

Donc, en tenant compte de la remarque 1.2, la formule (4) s'écrit

$$
\begin{aligned}
S_{1}\left(X^{(1)}\right) S_{1}\left(X^{(2)}\right) S_{2}\left(X^{(3)}\right) & =S_{1,1,2}\left(X^{(1)}, X^{(2)}, X^{(3)}\right) \\
& +S_{1,1,1}\left(X^{(1)}, X^{(3)}, X^{(2)} X^{(3)}\right) \\
& +S_{1,1,1}\left(X^{(2)}, X^{(3)}, X^{(1)} X^{(3)}\right) \\
& +S_{2,1}\left(X^{(3)}, X^{(1)} X^{(2)}\right) \\
& +S_{1,1}\left(X^{(1)} X^{(3)}, X^{(2)} X^{(3)}\right) \\
& +S_{1,1}\left(X^{(1)} X^{(2)} X^{(3)}, X^{(3)}\right)
\end{aligned}
$$

Remarque 2.4. - Les données du tableau ci-dessus ne dépendent pas du nombre $n$ d'indéterminées de chaque variable $\left(X^{(i)}\right)_{1 \leq i \leq 3}$. Cette décomposition de $S_{1}\left(X^{(1)}\right) S_{1}\left(X^{(2)}\right) S_{2}\left(X^{(3)}\right)$ est donc valable pour tout $n \geq 4$.

Lemme 2.5. - Soit $q, n \in \mathbb{N}^{*}$ et soit $X^{(\ell)}=\left(x_{1}^{(\ell)}, \ldots, x_{n}^{(\ell)}\right)$ pour $1 \leq \ell \leq q$, q ensembles de $n$ variables. Soit $\left(\alpha_{1}, \ldots, \alpha_{q}\right) \in \mathbb{N}^{q}$ tel que $\alpha_{1}+\cdots+\alpha_{q} \leq n$. Il existe un entier $s \geq 1$ tel que l'on ait :

$$
\begin{aligned}
\prod_{i=1}^{q} S_{\alpha_{i}}\left(X^{(i)}\right) & =S_{\alpha_{1}, \ldots, \alpha_{q}}\left(X^{(1)}, \ldots, X^{(q)}\right) \\
& +\sum_{k=1}^{s-1} S_{\beta_{1}, \ldots, \beta_{r_{k}}}\left(T^{(1)}, \ldots, T^{\left(r_{k}\right)}\right)
\end{aligned}
$$

où chaque $T^{(j)}$ est un monôme en les variables $X^{(i)}$ et chaque $\beta_{j}$ est un entier $\geq 1$ avec pour tout $k=1, \ldots, s-1, \beta_{1}+\cdots+\beta_{r_{k}}<\alpha_{1}+\cdots+\alpha_{q}$ et avec la convention $\sum_{\emptyset}=0$, ce qui correspond au cas éventuel $s=1$.

Démonstration. - On part de l'égalité (4). Soit $s$ le nombre de $N$-uplets $\left(j_{\sigma}\right)_{\sigma \in \mathcal{P}}$ intervenant dans la somme de l'égalité (4) i.e. le nombre de $N$-uplets vérifiant la condition (3). On numérote ces $N$-uplets de 1 à $s$. Pour tout entier $k$ tel que $1 \leq k \leq s$, on note $\beta_{1}, \ldots, \beta_{r_{k}}$ les entiers $j_{\sigma} \neq 0$ du $k$-ième $N$-uplet $\left(j_{\sigma}\right)_{\sigma \in \mathcal{P}}$ et on note aussi $T^{(1)}, \ldots, T^{\left(r_{k}\right)}$ les monômes $M_{\sigma}$ correspondants. L'égalité (4) s'écrit alors

$$
\prod_{i=1}^{q} S_{\alpha_{i}}\left(X^{(i)}\right)=\sum_{k=1}^{s} S_{\beta_{1}, \ldots, \beta_{r_{k}}}\left(T^{(1)}, \ldots, T^{\left(r_{k}\right)}\right)
$$


Tous ces $r_{k}$-uplets $\left(\beta_{1}, \ldots, \beta_{r_{k}}\right)$, pour $k=1, \ldots, s$, vérifient l'inégalité

$$
\sum_{\nu=1}^{r_{k}} \beta_{\nu} \leq \sum_{i=1}^{q} \alpha_{i}
$$

et l'égalité est atteinte pour un seul d'entre eux. En effet, puisque pour tout $\sigma \in \mathcal{P}$ on a Card $(\sigma) \geq 1$ alors

$$
\sum_{\nu=1}^{r_{k}} \beta_{\nu}=\sum_{\sigma \in \mathcal{P}} j_{\sigma} \leq \sum_{\sigma \in \mathcal{P}}\left(j_{\sigma} \operatorname{Card} \sigma\right)=\sum_{i=1}^{q}\left(\sum_{\substack{\sigma \in \mathcal{P} \\ i \in \sigma}} j_{\sigma}\right)=\sum_{i=1}^{q} \alpha_{i}
$$

et pour avoir l'égalité

$$
\sum_{\sigma \in \mathcal{P}} j_{\sigma}=\sum_{\sigma \in \mathcal{P}}\left(j_{\sigma} \operatorname{Card} \sigma\right)
$$

il faut et il suffit que pour tout $\sigma \in \mathcal{P}$ tel que $j_{\sigma} \neq 0$ on ait Card $\sigma=1$. Auquel cas les seuls $j_{\sigma}$ non nuls seront ceux pour lesquels $\sigma=\{i\}$ avec $i=1, \ldots, q$. Quitte à renuméroter les $N$-uplets $\left(j_{\sigma}\right)$, on peut supposer que celui-ci est le $s$-ième. On a donc $r_{s}=q$ et en vertu de la condition (3),

et par suite

$$
\left\{\begin{array}{l}
\beta_{i}=\alpha_{i}=j_{\{i\}} \text { pour tout } i=1, \ldots, q \\
T^{(i)}=X^{(i)}=M_{\{i\}} \text { pour tout } i=1, \ldots, q
\end{array}\right.
$$

$$
S_{\beta_{1}, \ldots, \beta_{r_{s}}}\left(T^{(1)}, \ldots, T^{\left(r_{s}\right)}\right)=S_{\alpha_{1}, \ldots, \alpha_{q}}\left(X^{(1)}, \ldots, X^{(q)}\right)
$$

Ainsi l'égalité (4) s'écrit sous la forme souhaitée en (5).

\section{Polarisation}

Dans ce paragraphe on utilise les lemmes $2.1,2.2$, et 2.5 pour démontrer le résultat principal annoncé en introduction, et l'illustrer à l'aide d'un exemple.

Théorème 3.1. - Soit $q, n \in \mathbb{N}^{*}$ et soit $X^{(\ell)}=\left(x_{1}^{(\ell)}, \ldots, x_{n}^{(\ell)}\right)$ pour $1 \leq \ell \leq q$, q ensembles de $n$ variables. Pour tout entier $j \leq n$, la $j$-ième fonction symétrique élémentaire

$$
S_{j}\left(X^{(1)}+\cdots+X^{(q)}\right)
$$

s'exprime comme polynôme à coefficients entiers en les fonctions symétriques $\left(S_{k}\right)$ pour $k \leq j$ et où les variables des $S_{k}$ sont des monômes de la forme

$$
\left(X^{(1)}\right)^{r_{1}} \cdots\left(X^{(q)}\right)^{r_{q}}
$$

pour certains entiers $r_{1}, \ldots, r_{q}$. Autrement dit

$$
S_{j}\left(X^{(1)}+\cdots+X^{(q)}\right) \in \mathbb{Z}\left[S_{k}\left(\left(X^{(1)}\right)^{r_{1}} \cdots\left(X^{(q)}\right)^{r_{q}}\right)\right]_{k \leq j}
$$

Démonstration. - Par la décomposition (2), il suffit de montrer que pour tout entier fixé $j \leq n$ et pour tout $\alpha=\left(\alpha_{1}, \ldots, \alpha_{q}\right) \in \mathbb{N}^{q}$ tel que $\alpha_{1}+\cdots \alpha_{q}=j$, on a

$$
S_{\alpha_{1}, \ldots, \alpha_{q}}\left(X^{(1)}, \ldots, X^{(q)}\right) \in \mathbb{Z}\left[S_{k}\left(\left(X^{(1)}\right)^{r_{1}} \cdots\left(X^{(q)}\right)^{r_{q}}\right)\right]_{k \leq j}
$$

En utilisant l'égalité (5) on montre (7) par récurrence sur $|\alpha|=\alpha_{1}+\cdots \alpha_{q}$. 
Pour $|\alpha|=1$, la propriété (7) est évidente $\left(S_{\alpha_{1}}\left(X^{(1)}\right) \in \mathbb{Z}\left[S_{\alpha_{1}}\left(X^{(1)}\right]\right)\right.$. Pour $|\alpha|>1$, l'hypothèse de récurrence permet d'affirmer, puisque $\beta_{1}+\cdots+\beta_{r_{k}}<|\alpha|$, que la propriété (7) est vraie pour

$$
\sum_{k=1}^{s-1} S_{\beta_{1}, \ldots, \beta_{r_{k}}}\left(T^{(1)}, \ldots, T^{\left(r_{k}\right)}\right) .
$$

La propriété (7) est vérifiée par chacune des fonction $S_{\alpha_{i}}\left(X^{(i)}\right)$ donc par le produit $\prod_{i=1}^{q} S_{\alpha_{i}}\left(X^{(i)}\right)$. Enfin, comme tout monôme en les variables $T^{(m)}$ est un monôme en les variables $X^{(i)}$ alors la propriété (7) est vérifiée par

$$
S_{\alpha_{1}, \ldots, \alpha_{q}}\left(X^{(1)}, \ldots, X^{(q)}\right)=\prod_{i=1}^{q} S_{\alpha_{i}}\left(X^{(i)}\right)-\sum_{k=1}^{s-1} S_{\beta_{1}, \ldots, \beta_{r_{k}}}\left(T^{(1)}, \ldots, T^{\left(r_{k}\right)}\right),
$$

ce qui achève la preuve du théorème.

Exemple 3.2. - Pour $q=2$ et $n \geq 4$. On pose, pour simplifier les notations, $X^{(1)}=X$ et $X^{(2)}=Y$ des variables à au moins 4 indéterminées. En appliquant (2) avec $j=4$, on a

$$
S_{4}(X+Y)=S_{4,0}(X, Y)+S_{0,4}(X, Y)+S_{3,1}(X, Y)+S_{1,3}(X, Y)+S_{2,2}(X, Y) .
$$

Et en appliquant (4) on a successivement

$$
\begin{aligned}
S_{3,1}(X, Y) & =S_{3}(X) S_{1}(Y)-S_{2,1}(X, X Y) \\
S_{1,3}(X, Y) & =S_{3,1}(Y, X)=S_{3}(Y) S_{1}(X)-S_{2,1}(Y, X Y) \\
S_{2,2}(X, Y) & =S_{2}(X) S_{2}(Y)-S_{1,1,1}(X, Y, X Y)-S_{2}(X Y) \\
S_{1,1}(X, Y) & =S_{1}(X) S_{1}(Y)-S_{1}(X Y) \\
S_{2,1}(X, Y) & =S_{2}(X) S_{1}(Y)-S_{1,1}(X, X Y) \\
& =S_{2}(X) S_{1}(Y)-S_{1}(X) S_{1}(Y)+S_{1}(X Y) .
\end{aligned}
$$

Donc

$$
\begin{aligned}
S_{2,1}(Y, X Y) & =S_{2}(Y) S_{1}(X Y)-S_{1}(Y) S_{1}(X Y)+S_{1}\left(X Y^{2}\right) \\
S_{2,1}(X, X Y) & =S_{2}(X) S_{1}(X Y)-S_{1}(X) S_{1}(X Y)+S_{1}\left(X^{2} Y\right) .
\end{aligned}
$$

De même, en appliquant (4) (avec $q=3$ ) on a

$$
\begin{aligned}
S_{1,1,1}(X, Y, Z) & =S_{1}(X) S_{1}(Y) S_{1}(Z)-S_{1,1}(X, Y Z) \\
& -S_{1,1}(Z, X Y)-S_{1,1}(Y, X Z)-S_{1}(X Y Z) \\
& =S_{1}(X) S_{1}(Y) S_{1}(Z)-S_{1}(X) S_{1}(Y Z)+S_{1}(X Y Z) \\
& -S_{1}(Z) S_{1}(X Y)+S_{1}(Z X Y)-S_{1}(Y) S_{1}(X Z) \\
& +S_{1}(Y X Z)-S_{1}(X Y Z) \\
& =S_{1}(X) S_{1}(Y) S_{1}(Z)-S_{1}(X) S_{1}(Y Z) \\
& -S_{1}(Z) S_{1}(X Y)-S_{1}(Y) S_{1}(X Z)+2 S_{1}(X Y Z) .
\end{aligned}
$$


Donc

$$
\begin{aligned}
S_{1,1,1}(X, Y, X Y) & =S_{1}(X) S_{1}(Y) S_{1}(X Y) \\
& -S_{1}(X) S_{1}\left(X Y^{2}\right)-S_{1}(Y) S_{1}\left(X^{2} Y\right) \\
& -S_{1}(X Y) S_{1}(X Y)+2 S_{1}\left(X^{2} Y^{2}\right)
\end{aligned}
$$

D'où finalement

$$
\begin{aligned}
S_{4,0}(X, Y) & =S_{4}(X) \\
S_{0,4}(X, Y) & =S_{4}(Y) \\
S_{3,1}(X, Y) & =S_{3}(X) S_{1}(Y)-S_{2}(X) S_{1}(X Y)+S_{1}(X) S_{1}(X Y)-S_{1}\left(X^{2} Y\right) \\
S_{1,3}(X, Y) & =S_{3}(Y) S_{1}(X)-S_{2}(Y) S_{1}(X Y)+S_{1}(Y) S_{1}(X Y)-S_{1}\left(X Y^{2}\right) \\
S_{2,2}(X, Y) & =S_{2}(X) S_{2}(Y)-S_{2}(X Y)-S_{1}(X) S_{1}(Y) S_{1}(X Y) \\
& +S_{1}(X) S_{1}\left(X Y^{2}\right)+S_{1}(Y) S_{1}\left(X^{2} Y\right)+S_{1}(X Y) S_{1}(X Y)-2 S_{1}\left(X^{2} Y^{2}\right)
\end{aligned}
$$

et

$$
\begin{aligned}
S_{4}(X+Y) & =S_{4}(X)+S_{4}(Y) \\
& +S_{3}(X) S_{1}(Y)-S_{2}(X) S_{1}(X Y)+S_{1}(X) S_{1}(X Y)-S_{1}\left(X^{2} Y\right) \\
& +S_{3}(Y) S_{1}(X)-S_{2}(Y) S_{1}(X Y)+S_{1}(Y) S_{1}(X Y)-S_{1}\left(X Y^{2}\right) \\
& +S_{2}(X) S_{2}(Y)-S_{2}(X Y)-S_{1}(X) S_{1}(Y) S_{1}(X Y) \\
& +S_{1}(X) S_{1}\left(X Y^{2}\right)+S_{1}(Y) S_{1}\left(X^{2} Y\right)+S_{1}(X Y) S_{1}(X Y)-2 S_{1}\left(X^{2} Y^{2}\right) \\
& \in \mathbb{Z}\left[S_{i}(X), S_{i}(Y), S_{1}(X Y), S_{1}\left(X Y^{2}\right), S_{1}\left(X^{2} Y\right), S_{1}\left(X^{2} Y^{2}\right), S_{2}(X Y)\right]_{i \leq 4}
\end{aligned}
$$

\section{Références}

[BeMé1] Bertin, J., Mézard, A. : Problem of formation of quotients and base change, Manuscripta Math. 115, 467-487 (2004).

[BeMé2] Bertin, J., Mézard, A. : Déformations formelles des revêtements sauvagement ramifiés de courbes algébriques, Invent. Math. 41, 195-238, (2000).

[DeGa] Demazure, M., Gabriel, P. : Groupes algébriques. Tome 1, Masson, (1970).

[Gi] Gillet, H. : Intersection theory on Algebraic stacks and Q-varieties, J. Pure. Appl. Alg. 34, 193240 (1984).

[LøKl] Lønsted, K., Kleiman, S. : Basics on families of hyperelliptic curves, Compositio Mathematica 38, no 1, 83-111 (1979).

[Ma] Macdonald, I.G. : Symmetric Functions and Hall polynomials, Clarendon Press, Second edition, Oxford (1995)

[Ra] Raynaud, M. : p-groupes et réduction semi-stable des courbes. In The Grothendieck Festschrift Volume III, Birkhauser, 1990, pp. 179-197.

01 juin 2011 
Mohamed KrIR, Laboratoire de mathématiques de Versailles, UMR CNRS 8100, Université de Versailles saint Quentin, 78035 Versailles cedex, France

- E-mail : mohamed.krir@uvsq.fr 